\title{
空间可展开天线机构研究与展望*
}

\author{
刘荣强 ${ }^{1}$ 史 创 $^{1} \quad$ 郭宏伟 $^{1}$ 李冰岩 $^{1} \quad$ 田大可 $^{2}$ 邓宗全 $^{1}$ \\ (1. 哈尔滨工业大学机器人技术与系统国家重点实验室 哈尔滨 150001; \\ 2. 沈阳建筑大学机械工程学院 沈阳 110168)
}

\begin{abstract}
摘要: 空间可展开天线机构是近年来快速发展的研究方向之一, 是支撑大型空间天线的关键装备, 其发展趋势是高展收比、 高精度、高刚度、轻量化。经过近 30 年的发展, 空间可展开天线已获得大量实际应用, 随着天线的超大型化需求, 经典的 结构形式不易满足天线日益增长的性能要求, 近年来出现了以百米级尺度为目标的可展开天线机构形式, 如协同展开式天线、 环柱式天线、薄膜天线等。介绍空间可展开天线机构的典型结构形式、应用案例、最新的技术进展，对可展开天线机构的构 型设计、优化设计、动力学分析的研究现状进行了综述, 分析可展开天线机构研究当前存在的主要问题与发展趋势, 为开展 空间可展开天线机构研究提供借鉴与参考。
\end{abstract}

关键词: 可展开机构; 星载天线; 构型设计; 优化设计; 动力学分析 中图分类号: V474

\section{Review of Space Deployable Antenna Mechanisms}

\section{LIU Rongqiang ${ }^{1}$ SHI Chuang ${ }^{1}$ GUO Hongwei ${ }^{1} \quad$ LI Bingyan $^{1} \quad$ TIAN Dake $^{2}$ DENG Zongquan ${ }^{1}$}

(1. State Key Laboratory of Robotics and System, Harbin Institute of Technology, Harbin 150001;

2. School of Mechanical Engineering, Shenyang Jianzhu University, Shenyang 110168)

\begin{abstract}
Space deployable antenna mechanism is one of the rapid developing research directions in recent years as well as the key equipment to support the large space antenna, and it has the development trend of higher aspect ratio, higher precision, higher stiffness and less mass. After nearly 30 years of development, amount of space deployable antennas have been applied to practical tasks. But the classic structural forms are not preferable to satisfy the increasing antenna performance requirement any more as the ultra-large antenna have become the mainstream demand. There are various deployable antenna mechanism forms with the level scale of a hundred of meters in recent years, such as collaborative deployable antennas, hoop-column antennas and membrane antennas, etc. It is mainly introduced that the typical structural forms, application cases and latest technical progress of the space deployable antenna mechanism, reviewing its research status of configuration design, optimization design and dynamic analysis, analyzing its current main problems and development trend, and providing references for researches on it.
\end{abstract}

Key words: deployable structure; space-borne antenna; configuration design; optimization design; dynamic analysis

\section{0 前言}

空间可展开天线是工作在太空环境下的具有展 开功能的天线。由于火箭有效发射容积的限制, 大 型星载天线在发射时需要将天线收拢起来, 入轨后 展开并进入工作状态。空间可展开天线作为传递与 获取信息的核心装备, 在空间通信、军事侦察、对 地观测、导航、深空探测等领域已得到广泛应用。 近年来, 为提高卫星通信距离、通信容量和对地观 测分辨率, 要求天线口径越来越趋向大型化, 美国

* 国家自然科学基金资助项目 $(51675114,51835002,51575119) 。 20190505$ 收到初稿, 20191107 收到修改稿
的 Thuraya 卫星天线口径为 $12.25 \mathrm{~m}$, 美国的 Harris 卫星天线口径为 $18 \mathrm{~m}$, 日本的试验卫星 ETS-VIII 天线口径为 $19 \mathrm{~m} \times 17 \mathrm{~m}$, 有报道称美国的地球静止 轨道侦查卫星天线口径已达到 50 100 m。

空间可展开天线可分为反射面天线和平面天线 两大类，反射面天线又可分为抛物面和抛物柱面两 种形式, 大型发射面天线多采用网状反射面结构, 以减轻天线重量、缩小收拢体积, 平面天线多用于 合成孔径雷达(简称 SAR), 是一种多波束天线。天 线可展开机构是实现天线收拢与展开功能、保证天 线型面精度和工作稳定性的重要装置, 目前常用的 可展开天线机构形式有环形析架式、单元构架式、 折叠肋式、缠绕肋式、索杆张拉式、环柱式、柔性 
自回弹式等。可展开机构作为天线骨架, 占据天线 质量的主要部分, 直接决定了天线结构的刚度和固 有频率, 除具有展收功能外, 还要求具有质量轻、 展开刚度和展开精度高等性能, 尤其是天线工作在 空间高真空与温度交变环境下, 对天线机构的展开 可靠性和热稳定性也提出了很高的要求。

近年来, 空间可展开天线机构已成为国内外的 研究热点, 主要集中在可展开机构构型设计、展开 驱动与控制、动力学特性分析、精度分析与补偿方 法、结构优化设计、地面试验与测试方法等方面, 随着复合材料、智能材料、薄膜材料等新材料的应 用, 将推动空间可展开机构向更大、更轻、更精的 方向发展, 需要将机械、材料、控制、力学、空间 物理学等多个学科进行交叉融合, 促进空间可展开 机构研究水平的提升和工程化应用。

\section{1 典型结构形式与应用案例}

可展开天线因应用背景和功能要求不同而具有 丰富的应用形式，不同类型的天线对于空间任务具 有不同的可适应性。国内外相关专著 ${ }^{[1-3]}$ 及论文对可 展开天线的典型结构形式与应用案例研究较多, 在 此仅对其内容进行简要介绍。

\section{1 环形天线}

环形天线是由可展环形杵架构成天线外围, 内 部由具有预应力的索网系统组成抛物面形状, 并由 镀金钼丝反射网接收与反射馈源信号 ${ }^{[4-5]}$ 。环形桁架 是由一系列平行四边形机构同步展开而形成的结 构, 环形天线的结构形式适应不同的口径要求, 其 质量不会随面积的增大而成比例增长 ${ }^{[6-7]}$, 且具有收 拢体积小、结构形式简单的优点。从 2000 年到 2007 年之间, 共有 5 个 AstroMesh 环形天线分别在 3 个 型号的航天器上实现在轨应用, 口径均为 $12.5 \mathrm{~m}$ 。

\section{2 构架式天线}

构架式可展开天线为反射面提供了全桁架式支 撑, 整体具有更高的刚度, 同时也兼具较大的折展 比。俄罗斯 Georgian 公司设计的 EGS 可展开天线 是一种剪叉式构架, 形面精度可以达到 $2.1 \mathrm{~mm}$, 该 构架式天线由中心向周边进行伞状展开 ${ }^{[8-10]}$ 。2006 年, 日本 Japan Aerospace Wxploration Agency (JAXA) 在卫星 Engineering Test Satellite VIII (ETS-VIII) 上进行了空间模块化构架式天线的展开。该构架 式天线系统包含两个反射面, 分别由 14 个口径为 $4.8 \mathrm{~m}$ 六边形可展开桁架模块组成, 每个展开尺寸为 $19.2 \mathrm{~m} \times 16.7 \mathrm{~m}$ (天线口径 $13 \mathrm{~m}$ ), 收拢尺寸为 $1 \mathrm{~m}$ (直 径) $\times 4 \mathrm{~m}$ (高度), 形面精度为 $2.4 \mathrm{~mm}$, 系统质量为
$250 \mathrm{~kg}^{[11-13]}$ 。俄罗斯的 OKB-MEI 公司研制了四面体 构架式天线, 它由分布于其中大量弹簧进行驱动, 但在展开结束时仍会发现有些铰链没有完全展 开 $^{[14]}$ 。天线的口径可以通过改变模块的数量和大小 来适应不同的任务需求。

\section{3 平面天线}

平面天线可以提供更强的广角波束扫描能力。 合成孔径雷达(简称 SAR)天线是平面天线的典型应 用案例, 美国国家航空航天局(NASA)喷气推进实验 室(JPL)于 1978 年发射了世界首颗平面 SAR 天线 Seasat, 分辨率为 $25 \mathrm{~m}^{[15]}$ 。欧空局于 1991 年和 1995 年分别发射了民用雷达 ERS-1 和 ERS-2, 于 2002 年发射地球环境检测卫星 ENVISAT, 均具备 $30 \mathrm{~m}$ 的空间分辨率 ${ }^{[16-17]}$ 。加拿大航天局分别于 1995 年和 2007 年发射了 Radarsat-1 和 Radarsat-2 卫星, 其中, Radarsat- 1 最大分辨率为 $8 \mathrm{~m}$, 而 Radarsat- 2 的分辨 率可实现从 $3 \mathrm{~m}$ 到 $100 \mathrm{~m}$ 之间可选成像 ${ }^{[18-19]}$ 。日本 于 2006 年发射了对地观测卫星 ALOS, 最大分辨率 可达 $2.5 \mathrm{~m}^{[20]}$ 。该类天线以可展开桁架或者驱动铰 链推动天线面板展开, 具有展开运动简单、展开刚 度高的特点。

\section{4 肋式天线}

肋式天线采用多根抛物线形肋条对反射面进行 支撑，进行伞状形式展开。美国 Harris 公司研制了 径向肋天线,应用于 NASA 的 Tracking \& Data Relay Satellite(TDRS)项目上, 自 1983 年以来共成功应用 12 次。由于采用刚性肋支撑, 轴向无法折叠, 导致 收拢高度过大, 针对此问题 Harris 公司提出折叠肋 方案, 通过在肋上设置铰链, 实现肋的多段折叠, 从而减小收拢体积, 并于 2000 年在 ACES 航天器 的 Garuda 移动通信系统上成功完成了 $12 \mathrm{~m}$ 口径天 线的展开。NASA 还开发出收拢体积更小的缠绕肋 天线, 收拢时肋缠绕在中心轮毂上, 并由绳索将其 锁紧; 展开时, 肋依靠变形时穴存的弹性势能而反 向打开, 恢复至初始状态时天线完全展开, 其口径 为 $9.1 \mathrm{~m}$ 。日本宇航局在 1997 年发射的天文卫星 HALCA 上展开了口径为 $8 \mathrm{~m}$ 的肋式天线, 其 6 根 肋为伸展臂, 用于对抛物网面的支撑 ${ }^{[21-22] 。}$

\section{5 张拉式天线}

张拉式可展开天线充分利用了绳索的单向受 拉和易收拢的特性, 大幅降低了可展天线的质量, 美国 Harris 公司将张拉式天线于 2009 年成功应用 于 TerreStar-1 卫星上 ${ }^{[23]}$ 。TerreStar-1 天线主要由 金属反射面和索胁刚化折展机构组成。索肋刚化 折展机构由径向胁、柔性索、关节铰链、中心支 撑杆、同步联动机构、驱动装置等组成。6 根径向 
肋在圆周方向均匀布置、呈辐射状, 中心处共同 连接在中心支撑杆上, 使得各肋处于连续、非孤 立状态以提高结构刚度。径向肋外端节点之间通 过柔性索进行张紧连接, 使展开后的径向肋处在
固定的空间位置, 构成索肋系统, 对天线网面起 到牢固支撑作用。

综上, 目前典型在轨成功应用的可展开天线如 表 1 所示。

表 1 空间可展开天线典型应用

\begin{tabular}{|c|c|c|c|c|c|}
\hline 类型 & 项目名称 & 研究单位 & 发射时间 & 口径/m & 质量 $/ \mathrm{kg}$ \\
\hline \multirow{3}{*}{ 环形 } & Thuraya & Astro Aerospace & 2000,2003 & 12.25 & 55 \\
\hline & MBSat & Astro Aerospace & 2004 & 12 & - \\
\hline & Inmarsat 4 & Astro Aerospace & 2005 & 9 & \\
\hline \multirow{2}{*}{ 构架 } & EGS & 俄罗斯 Georgian 公司 & 1999 & 7 & - \\
\hline & ETS-VIII & 日本 JAXA & 2002 & 13 & 340 \\
\hline \multirow{7}{*}{ 平面 } & Seasat & JPL & 1978 & $10.7 \times 2.2$ & 59 \\
\hline & ERS-1 & 欧空局 & 1991 & $10 \times 1$ & 150 \\
\hline & ERS-2 & 欧空局 & 1995 & - & 150 \\
\hline & ENVISAT & 欧空局 & 2002 & $10 \times 1.3$ & 600 \\
\hline & Radarsat-1 & 加拿大宇航局 & 1995 & $15 \times 1.5$ & 446 \\
\hline & Radarsat-2 & 加拿大宇航局 & 2007 & $15 \times 1.4$ & 440 \\
\hline & ALOS & JAXA & 2006 & $8.9 \times 3.1$ & 600 \\
\hline \multirow{5}{*}{ 肋式 } & HALCA & JAXA & 1997 & 8 & 246 \\
\hline & MUOS-5 & Harris 公司 & 2016 & 14 & - \\
\hline & ATS-6 & NASA & 1974 & 9.1 & 60 \\
\hline & TDRS & Harris 公司 & 1983 & 4.9 & - \\
\hline & ACES & Harris 公司 & 2000 & 12 & - \\
\hline $\begin{array}{c}\text { Harris 张拉式 } \\
\text { 天线 }\end{array}$ & TerreStar-1 & Harris 公司 & 2009 & 18 & - \\
\hline
\end{tabular}

\section{2 可展开天线机构技术最新进展}

随着航天事业的快速发展, 可展开天线口径越 来越趋向于大型化, 天线口径或机构展开尺寸达到 数十米甚至百米。在天线大型化的同时, 需要解决 轻量化和大展收比的问题, 同时要满足天线的刚度 和精度要求。目前国际上在百米级超大型天线、薄 膜天线等方面的研究取得了突破性进展, 成为未来 空间可展开天线发展的趋势。

\section{1 协同展开式抛物柱面天线}

2011 年, 美国 DARPA 资助的 Innovative Space Based Radar Antenna Technology(ISAT)计划研制一 种大型抛物柱面天线 ${ }^{[24-25]}$, 如图 1 所示。其展开原 理是利用一维伸展臂机构带动网面和天线馈源实现
伸展臂与天线协同展开。在网面支架之间增加预紧 绳索使整个结构在大跨度上能够保持稳定平衡, 并 降低伸展臂的负载压力, 提高整体刚度。

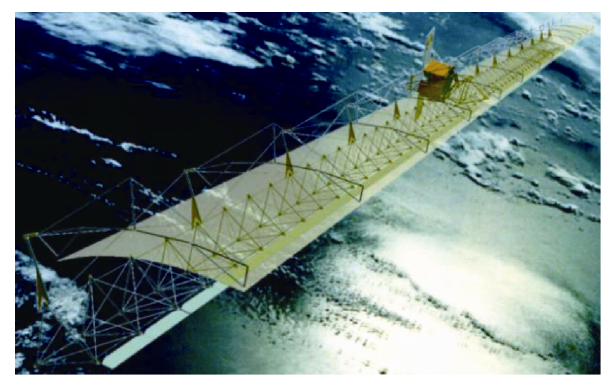

图 1 ISAT 效果图

该天线的设计长度为 $108 \mathrm{~m}$, 目前已制作出 12 $\mathrm{m} \times 6 \mathrm{~m}$ 的天线样机, 它包含一个 $12 \mathrm{~m}$ 可展开支撑 臂, 其展开过程如图 2 所示。为了实现大折叠比展

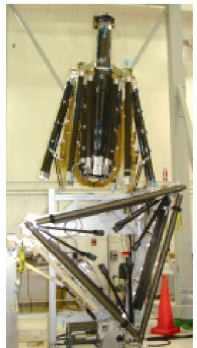

(a) 收拢状态

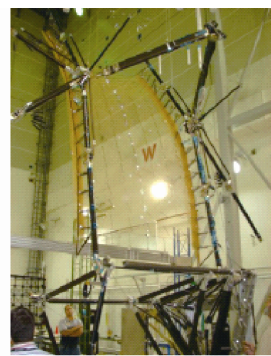

(b) 展开过程

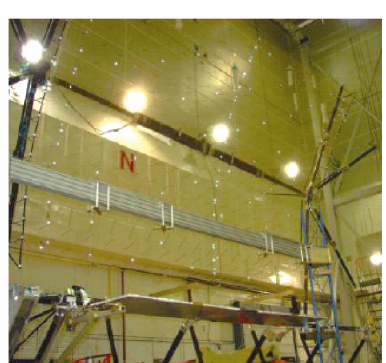

(c) 展开状态

图 2 ISAT 桁架式抛物柱面天线展开过程 
开, 设计了可折叠丝杜, 单元之间采用弹性铰链进 行展开驱动, 实现展开驱动与锁定的一体化设计。

\section{2 大型环柱式天线}

美国 Harris 公司研制了 $15 \mathrm{~m}$ 口径的环柱式 (Maypole Hoop and Column)可展开天线机构, 并进 行了相应的地面测试和在轨展开试验。如图 3 所示, 环柱形可展开天线由环胁、中央伸缩圆柱、张紧拉 索与金属反射网构成。

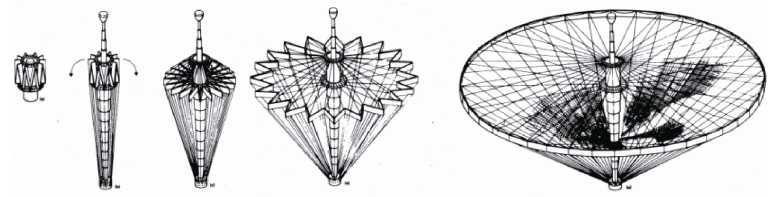

图 3 环柱状天线机构展收过程

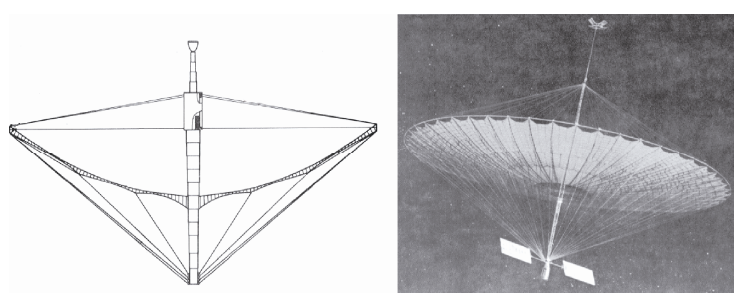

图 4 环柱式天线结构组成示意图与实物图

天线展开初期, 环胁、张紧拉索与金属反射网 折叠收拢于中央伸缩圆柱的表面, 随着中央伸缩圆 柱的伸长, 环肋呈径射状展开, 最终完成整个机构 的展开 ${ }^{[26]}$ 。环柱式天线的中心立柱与天线外缘析架 间通过上下两层布置的张力索相连, 形成了空间平 衡力系, 对天线起到支撑作用, 提高了天线的整体 刚度, 且具有收拢体积小、质量轻的特点, 因此适 合于大型化天线, 目前美国的在轨应用环柱式天线 口径估计为 $50 \sim 100 \mathrm{~m}^{[26]}$ 。

\section{3 双层环形天线}

俄罗斯 Georgian 公司设计了一种剪叉式双层可 展开天线 ${ }^{[27]}$, 该天线机构的外层可展开环形桁架由 剪叉机构组成, 使用辐射状的张拉膜肋连接外层支 撑桁架与中央轮毂, 实现整个天线机构刚度的提升。 其收拢与展开状态如图 5 所示。后续还研制了口径 为 $30 \mathrm{~m}$ 的天线机构样机, 如图 6 所示。

双层环形结构可实现天线的大口径, 据报道目 前在轨应用的大口径天线有直径为 $90 \mathrm{~m}$ 的美国“喇 叭” (TRUMPET) 可展开天线、直径为 $105 \mathrm{~m}$ 的 “水
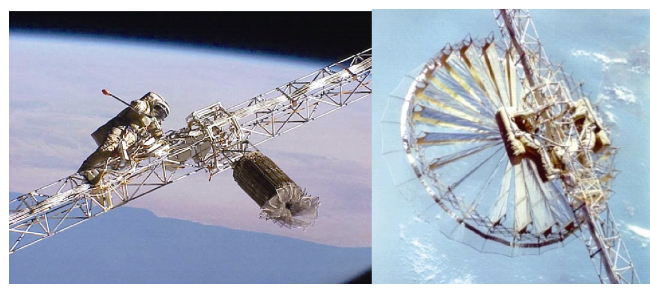

图 5 EGS 展开天线

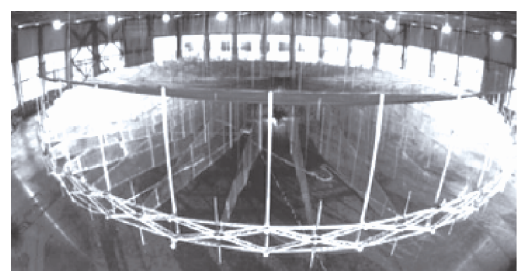

图 $630 \mathrm{mEGS}$ 可展开天线机构

星” (MERCURY) 可展开天线、直径为 $150 \mathrm{~m}$ 的 “先 进折叠座椅” (Jumpseat) 可展开天线, 均为可展开双 层环形析架结构, 如图 7 所示。
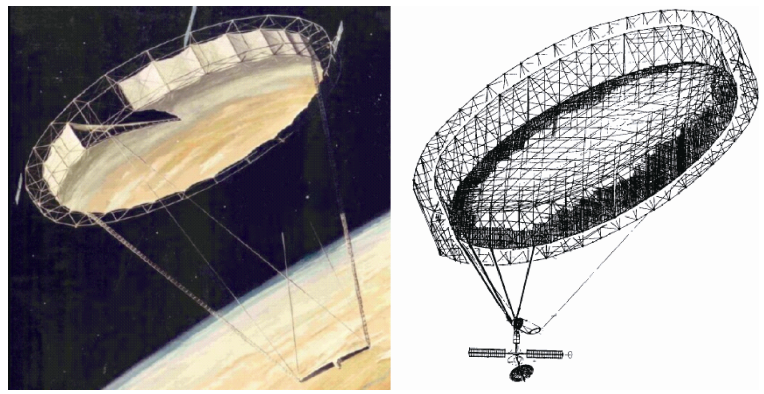

图 7 双层可展开天线机构

此外, 浙江大学空间结构研究中心关富玲教授等 设计了双层环形析架式可展开天线原理样机 ${ }^{[28-29]}$ 。 GILGER 等 ${ }^{[30]}$ 提出了一种索杆张拉式双环可展开天 线机构, 其内层为环形可展开桁架机构, 外层通过 伸杆通过绳索与内层桁架张拉, 从而提高了整个天 线机构的刚度。

\section{4 薄膜天线与薄膜光学系统}

美国喷气推进实验室(JPL)于 1997 年研制的薄 膜天线如图 8 所示 ${ }^{[31]}$ 。样机尺寸 $3.3 \mathrm{~m} \times 1.0 \mathrm{~m}$, 天线 阵面采用三层薄膜结构, 薄膜材料由 $0.13 \mathrm{~mm}$ 厚的 Kapton 介质材料构成, 涂敷的铜层厚度为 $5 \mu \mathrm{m}$ 。薄 膜与周边支撑杆件由一系列的张力索连接。天线周 边桁架采用复合充气式材料结构, 天线阵面单位面 积质量为 $3.3 \mathrm{~kg} / \mathrm{m}^{2}$ 。

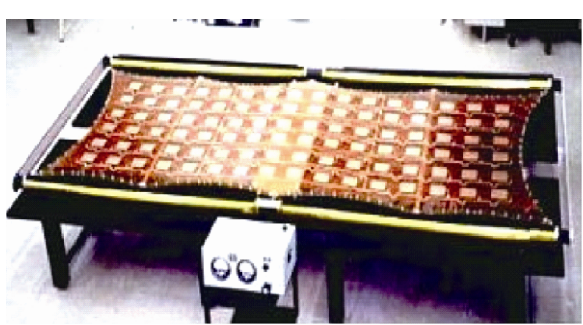

图 8 JPL 实验室开发的 L 波段薄膜阵列天线样机

德国 DLR 和欧空局合作研制了面积为 $40 \mathrm{~m}^{2}$ 、 总质量不超过 $60 \mathrm{~kg}$ 的薄膜天线, 如图 9 所示。采 用弹性材料伸杆(豆荚杆)作为 SAR 天线支架 ${ }^{[32]}$ 。

2010 年美国 DARPA 启动了 “MOIRE” 项目计 划, 计划在 2020 年左右发射地球静止轨道 $20 \mathrm{~m}$ 口 径望远镜, 实现对地 $24 \mathrm{~h}$ 实时监视侦察 ${ }^{[33]}$ 。其主镜 


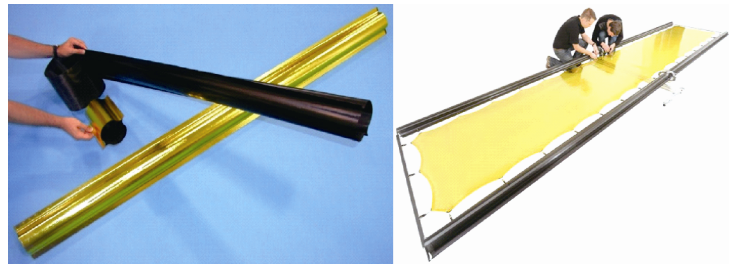

图 9 豆荚杆及可展开 SAR 天线样机

采用了环扇形的分块方式。主镜由 18 分块的子镜铰 接而成, 收拢时呈一个圆雉台形(图 10、11)。

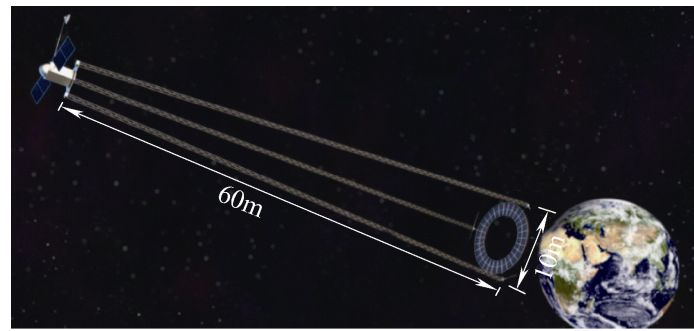

图 10 美国 “MOIRE” 计划薄膜光学系统示意图

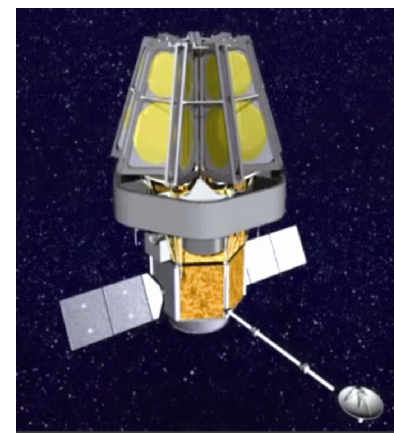

图 11 光学主镜扇形分块及收拢状态示意图

\section{5 充气展开式天线}

充气展开天线是一种通过充气膨胀至所需反射 面型面的薄膜结构天线, 具有质量轻、收拢体积小 的特点(图 12)。1996 年 5 月美国开展了充气展开天 线的在轨试验(IAE 试验), 该天线由 L'Garde 公司研 制, 口径为 $14 \mathrm{~m}$, 充气结构由充气式反射器组合装 置、支撑环和支撑杆三部分构成, 反射器的反射面 由 Mylar 膜构成, 支撑环和支撑杆由凯夫拉纤维聚 乙烯膜构成 ${ }^{[34]}$ 。

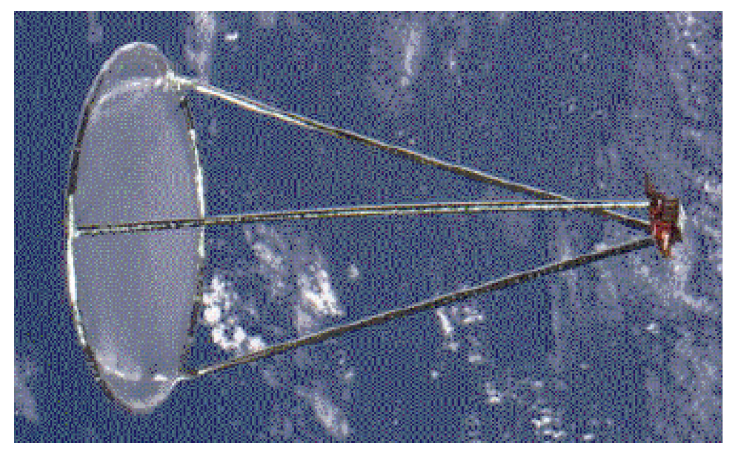

图 12 IAE 试验中的充气天线

美国 JPL 实验室研制了口径 $1 \mathrm{~m}$ 的 X 波段和口 径 $3 \mathrm{~m}$ 的 $\mathrm{Ka}$ 波段的微带反射阵列天线 ${ }^{[35]}$, 如图 13
所示。欧洲航天局联合 Contraves 公司开展了基于 ISRS 技术的充气空间自固化天线的研制, 其天线的 薄膜材料为树脂基的凯夫拉纤维, 依靠太阳照射来 完成展开及硬化 ${ }^{[35]}$, 如图 14 所示。SRS 公司为 NASA 研制了口径为 $35 \mathrm{~m}$ 由形状记忆复合材料制 成的大口径充气展开天线。加利福尼亚大学研制了 口径为 $25 \mathrm{~m}$ 的充气展开天线用于美国 ARISE 计划。

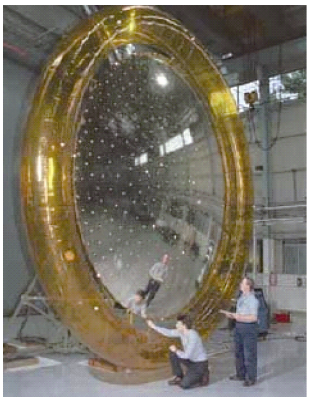

图 $13 \mathrm{JPL} \mathrm{Ka}$ 波段充气展开天线

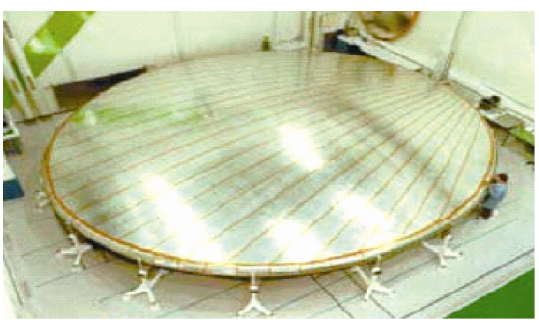

图 14 空间自固化天线

NASA 兰利研究中心在 2002 年研究了 Hexapod 充气天线, 如图 15 所示, 它是一种充气式可展薄膜 天线, 目标口径为 $100 \mathrm{~m}$, 它由多个小型充气天线 单元组合而成。每个单元的薄膜固定于圆环上, 圆 环由六根充气杆支撑, 形成一个三角形的孔径。天 线主体结构材料为可充气、可刚化的薄壁复合材料 管, 该材料可以在发射时存储在很小的空间内。为 了加强形状精度和振动控制, 膜与充气杆之间采用 绳索连接, 并增加了压电材料进行振动控制。

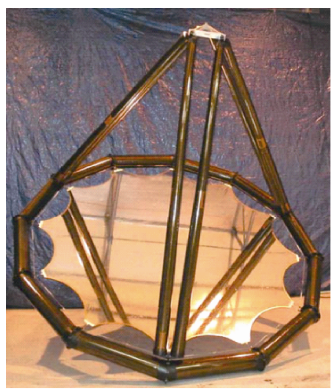

(a) 充气天线单

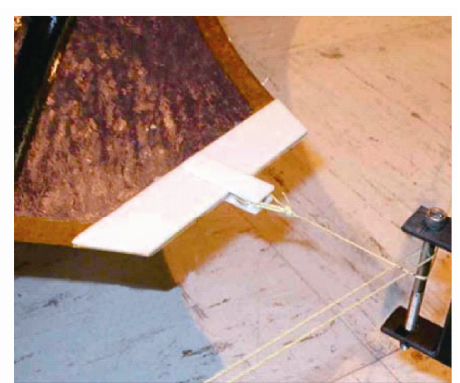

(b) 绳索预紧系统与薄膜边缘设计

图 15 Hexapod 充气天线

国内方面, 哈尔滨工业大学复合材料与结构研 究所的人员对充气天线膜结构的褶皱及振动特性和 空间充气管的展开过程进行了研究 ${ }^{[36-37]}$ 。浙江大学 徐彦等人对充气展天线反射面的型面精度开展了研 
究, 并设计制造了口径为 $2 \mathrm{~m}$ 的样机 ${ }^{[38]}$ 。2006 年, 上海航天技术研究所同上海交通大学、同济大学共 同研制了口径分别为 $1 \mathrm{~m}$ 和 $3 \mathrm{~m}$ 的抛物面充气可展 天线原理样机, 两个样机的反射面均是利用 Mylar 膜采用基于理想抛物面的分瓣裁切粘合而成, 反射 面通过裙边和预应力钢索与支撑环相连, 通过反射 面与裙边上的气嘴充气展开所需型面 ${ }^{[39]}$ 。

\section{3 可展开天线机构设计与分析现状}

\section{1 构型设计}

目前国际上对可展开机构新构型的设计, 主要 是通过对已有机构单元进行组网, 进而得到可展开 机构新构型。根据所用的单元不同, 这些构型主要 可以分为平面机构和空间机构两类。

平面机构中最典型的就是以剪刀机构及其变形 机构作为单元进行组网, 是最早研究也是最为成熟 的可展开机构构型设计方法。YOU 等 ${ }^{[40]}$ 利用平面剪 刀机构设计了一系列平面可展开机构网络, 并对其 构成的直线和曲线周长环的几何条件进行了研究, 进而派生出一些多边形和圆形周长环的放射型可展 开机构。KASSABIAN 等 ${ }^{[41]}$ 利用剪刀机构设计了一 种可展开屋顶, 并给出了该机构的几何约束条件。

PATEL 等 ${ }^{[42]}$ 对剪刀变形机构运动轨迹求解, 进而提 出了一种平面放射可展开机构的运动学分析方法。

TANAKA 等 ${ }^{[43]}$ 提出了一种用 8 杆剪刀机构组成平 面放射可展开机构网络的方法。

WARNAAR 等 ${ }^{[44-45]}$ 借助于图论对空间可展开 机构进行了系统的综合, 建立了三棱柱单元和八面 体单元的构型库。WOHLHART ${ }^{[46]}$ 通过在规则多面 体面上插入平面连杆的方法, 设计出一系列的多面 体过约束连杆可展开机构。KOVACS 等 ${ }^{[47]}$ 提出了一 系列的由棱柱体作为铰接平面的多面体可展开机 构, 并用数值模拟的方法对该类机构进行了运动分 析。HOBERMAN ${ }^{[48]}$ 提出了一种以雉齿轮传动的单 自由度多面体可展开机构。GOSSELIN 等 ${ }^{[49]}$ 以平面 单自由度多边形连杆为单元, 通过替代多面体表面 的方法, 构建出了一系列的多面体可展开机构。 KIPER 等 ${ }^{[50]}$ 基于万向接头对多面体可展开机构进 行了综合, 得出一些新型多面体可展开机构, 并用 单自由度平面连杆机构, 构造出一系列的多边形和 多面体可展开机构 ${ }^{[51]}$ 。

空间机构构型研究目前主要集中在过约束闭环 机构。2005 年, YOU 等 ${ }^{[52-59]}$ 采用单环过约束空间 机构作为单元, 实现可展开机构构型设计和组网。 他们对 Bennett 机构、Myard 机构和 Bricard 机构进
行了研究和改进, 得到了大量的空间闭环过约束可 折展机构, 并对它们的组网、奇异位形和运动学特 性展开了深入的研究。GAN 等 ${ }^{[60]}$ 提出了一种单闭环 可展开机构数值运动分析方法。BAKER ${ }^{[61]}$ 提出了多 种空间六杆可展开机构, 并对 Bennett 连杆做了深 入的运动学性能的探讨 ${ }^{[62]}$ 。VIQUERAT ${ }^{[63]}$ 基于 Bricard 机构设计了一种单自由度六杆平面可展开 机构, 对其机构原理进行了分析。YU 等 ${ }^{[64]}$ 基于 Bennett 连杆提出一种可支撑薄膜的可展开机构, 该 机构展开后可以将薄膜张紧成双曲面。

\section{2 优化设计}

众多国内外学者对可展开天线机构的优化设计 进行了大量的探索。2014 年 NAGASE 等 ${ }^{[65-67]}$ 提出 张拉整体结构基于力密度和连接矩阵的线性规划思 想, 在优化结构最小设计质量方面取得了突破性进 展。尤国强等以天线周边桁架壁厚和索单元预应力 为设计变量, 结构重量和反射面精度为优化目标, 结构基频为约束条件，建立了天线展开状态下的多 目标优化数学模型 ${ }^{[68]}$ 。李涁等 ${ }^{[69]}$ 建立了网状反射面 天线的两态优化模型, 对其进行优化设计, 开发了 基于环形展开桁架天线的多学科综合设计平台。罗 鹰等 ${ }^{[70]}$ 建立了周边桁架式可展开天线结构处于展 开状态时的动力优化模型。

2006 年, 美国 JPL 公司 FANG 等 ${ }^{[71]}$ 将双重索网 张拉系统应用到薄膜天线上, 以支撑框架受力最小为 目标对花边数量、跨距进行了优化。SAKAMOTO ${ }^{[72]}$ 理论上证明薄膜外形为圆弧状时膜内应力为最佳张 紧状态, 对索膜结构进行了优化设计。GRESCHIK 等 ${ }^{[73]}$ 研究了轴对称薄膜反射面参数对型面精度的 敏感性, 表明了褶皱引起的误差可以得到预计。 $\mathrm{SOH}$ 等 ${ }^{[74]}$ 采用了常数、线性、二次、高次函数拟合 薄膜厚度进行优化分析。2008 年, 关富玲等基于支 撑框架受力最小优化了双重索网张拉系统的薄膜花 边数量 ${ }^{[75]}$ 。

\section{3 动力学分析}

空间可展开天线机构一般多采用模块化设计, 包含大量的杆件、铰链、柔性索、驱动与锁定机构 等, 折展机构展开过程结构拓扑特征具有时变性、 结构刚度不断变化, 国内外对折展机构展开过程动 力学研究多采用多体动力学。

ROBERSON-WITTENBURG 提出了一种用于 分析多刚体系统的普遍方法, 简称为 $\mathrm{R} / \mathrm{W}$ 方法。 KANE 利用达朗贝尔原理直接建立动力学方程, 将 矢量形式的力和达朗贝尔惯性力直接向特定的单位 基矢量进行投影, 以消除约束力。SHABANA 等 ${ }^{[76]}$ 基于连续介质力学和有限元方法提出的绝对节点坐 
标法(ANCF) 是柔性多体系统的动力学发展的一个 里程碑。以 ANCF 方法建立的多柔体系统动力学方 程具有常数质量矩阵、不存在科氏力和离心力项等 特点, 比传统浮动坐标法、大转动矢量法等更能精 确地反映含超柔性构件的可展开空间结构的动力学 特性。

QUISENBERRY 等 ${ }^{[77] 、 P O O L E ~}$ 等 $^{[78]}$ 分别采用 有限段法、集中质量法等模拟了柔性索的运动过程。 MITSUGI 等 ${ }^{[79]}$ 建立了含柔性索网的星载抛物面桁 架天线机构多柔体动力学模型, 分析了机构展开过 程动力学特性。 NETO 等 ${ }^{[80]}$ 建立了基于复合材料板 和梁单元的欧洲 ERS-1 卫星的柔性太阳能电池阵 列、柔性桁架、刚性本体系统的多柔体模型, 并采 用模态综合法降低求解规模, 分析了柔性空间机构 与卫星本体之间的耦合动力学特性。任革学等 ${ }^{[1-82]}$ 基于绝对节点坐标法对含有刚性杆件、柔性索的空 间折展机构展开过程进行运动学和动力学的建模, 得出折展机构展开过程柔索和杆件的运动过程及规 律。蒋建平等 ${ }^{[83]}$ 对大挠性多体结构卫星刚柔耦合动 力学进行研究, 建立了系统的刚柔耦合一次近似动 力学方程, 得到了导致动力刚化现象的动力刚度项 并进行了数值仿真。刘钦鹏等 ${ }^{[84}$ 利用浮动坐标系进 行弹性构件建模, 利用拉格朗日方法对展开过程动 力学进行了分析。刘铖等 ${ }^{[85]}$ 发展了一种基于 $\mathrm{ANCF}$ 方法的复合材料层合板单元, 提出了一种基于 OpenMP 的高效并行计算方法, 研究了星载折叠式 太阳翼的展开动力学与控制问题。

综上所述, 虽然目前多体动力学建模方法研究 已经初步成熟, 但是对可展开机构这样的柔性多体 系统中柔性索非线性影响问题的研究才刚刚开始, 有待于进一步研究完善。

\section{4 地面模拟试验与验证}

可展开天线地面试验的内容包括: 重力环境模 拟、型面精度测试、动力学特性测试、可靠性试验 等, 具体如下。

(1) 重力环境模拟。目前微重力环境模拟的方法 主要有落塔法、抛物线飞行法、气浮法、水浮法和 悬吊法等 ${ }^{[86]}$ 。落塔法和抛物线飞行法提供的微重力 环境试验试验仅有 $3 \sim 30 \mathrm{~s}^{[87-88]}$, 水浮法 ${ }^{[89-90]}$ 受水流 阻力影响较大, 适用于可展开天线的微重力环境模 拟方法有悬吊法 ${ }^{[91-93]}$ 和气浮法 ${ }^{[94]}$, 目前, 美国 NASA 和日本 JAXA 等研究机构主要采用悬吊法进行可展 天线微重力模拟, 并研制了环形可展天线、空间伸 展臂和构架式可展天线等展开机构的悬吊系统。

(2) 天线型面精度测量。目前, 三维坐标测量 的仪器或方法主要有三坐标测量仪、全站仪、关节
臂和摄影测量系统等 ${ }^{[95-97]}$ 。三坐标测量仪操作简单、 使用方便，但被测试物体需固定在测试平台上; 全 站仪主要用于测试较远物体的角度、距离和坐标等; 关节臂体积小、轻便易携带, 可进行空间任意位置 的测量, 但仪器需要手持使用, 精度较低, 且单次 测量范围较小; 摄影测量系统利用交会测量原理, 通过单台或多台相机在不同的角度对被测物体进行 非接触测量, 具有测量精度高、携带方便、使用灵 活, 受环境影响小等优点。

(3) 动力学特性测试。可展天线的动力学环境 主要有振动环境、冲击环境和噪声环境, 为测试发 射时天线的抗振动冲击能力, 需要在天线收拢和压 紧状态下进行正弦振动、随机振动、冲击和噪声试 验等动力学试验 $[98]$ 。在展开状态下需测试天线的振 动模态和基频。国内外学者也对可展天线进行了相 关的动力学试验与研究。MISAWA 等 ${ }^{[99]}$ 对可展天线 发射前的频率进行了分析及试验验证，分析了模块 数量对天线频率的影响, 并对天线频率进行了预测。 ANDO 等 ${ }^{[100]}$ 对可展天线的网面及支撑桁架进行了 多柔体动力学分析, 开发了多柔体动力学软件 SPADE, 设计了原理样机并进行了试验研究。田大 可等 ${ }^{[101]}$ 开展了某构架式可展天线自由边界和中心 约束条件下的模态试验, 分析了结构参数对动力学 特性的影响。

(4) 可靠性试验。空间环境是一个高真空、大 温差、强辐射的苛刻环境, 容易引起关节润滑失效 卡死、构件热变形、材料性能退化等问题, 可展开 天线需在模拟空间环境下进行重复展收试验, 以检 验天线展开功能的可靠性, 防止在太空中出现展开 卡滞问题。由于天线展开后一般尺寸较大, 故在真 空罐中只进行局部或可展开单元的可靠性试验。

\section{4 存在的关键问题与发展趋势}

(1) 天线与卫星平台一体化设计。

随着天线向超大型化发展, 卫星平台尺度远小 于天线展开后尺度, 传统的以卫星平台为结构总体、 以天线为有效载荷的设计方法无法满足天线大型化 发展的需要, 天线已成为航天器的主结构, 卫星本 体作为服务平台, 需要为天线提供通信、导航、测 控、推进、能源等保障, 天线与卫星平台成为不可 分割的有机整体, 需要按照天线的功能要求导出卫 星平台的设计输入, 实现天线与卫星平台的一体化 设计。目前国内外尚无成熟的一体化设计方法。

（2）新材料应用与结构轻量化。

传统的可展开天线机构多为桁架式机构, 主要 
由刚性杆件与关节铰链构成, 采用电机控制, 随着 天线机构的大型化, 其本体质量急剧增大。天线结 构轻量化设计是亟需解决的关键问题之一。近年来 随着材料技术的发展, 新材料在天线机构中获得越 来越多的应用, 如用碳纤维复合材料替代普通金属 材料、用柔性索替代刚性构件、用智能复合材料制 作驱动元件替代电机驱动、用薄膜材料替代刚性天 线面板等。新材料应用大幅度降低了天线重量, 但 也导致了系统刚度的降低, 影响了在轨工作的稳定 度, 需通过多构态结构优化设计来提高系统刚度。

(3) 超大型复杂系统动力学及控制。

超大型天线机构在轨工作时是一个大柔性系 统, 系统的第一阶固有频率甚至低于 $0.1 \mathrm{~Hz}$, 其动 力学行为极其复杂, 而且天线机构由大量的杆、索、 网(或膜)组成, 存在大量柔性元件, 传统的有限元 动力学模型已不适用。近年来发展起来的绝对节点 坐标法虽然可以处理大柔性问题, 但还不能解决大 规模柔性结构的动力学分析问题, 在计算时间、计 算效率等方面难以满足要求。超大型复杂柔性系统 动力学建模与分析问题是天线机构的关键问题。另 外, 超大型天线机构对星体动力学与姿态控制产生 很大影响, 由于存在大柔性与大惯量问题, 给卫星 姿态控制和变轨调节带来很大挑战。

(4) 大柔性展开机构地面试验与测试。

目前二维重力补偿技术已较为成熟, 空间三维 重力补偿技术尚不完善, 尤其是大范围三维重力补 偿问题, 仍是国际上可展开天线面临的难题之一。 另一方面, 对于大柔性展开机构, 一般采用摄影测 量系统进行型面精度测量, 现有的非接触式激光测 振仪无法完成全尺寸样机的振动模态测试, 需要采 用缩小比例的或局部性样机来进行地面测试, 再通 过仿真软件和地面测试数据来预示全尺寸天线机构 在轨状态下的工作性能参数。建立大型天线机构的 虚拟仿真与地面实物测试的天地一体化验证系统十 分必要。

\section{5 结论}

本文介绍了空间可展开天线机构的典型结构形 式、应用案例、发展趋势及最新的技术进展, 对协 同展开式抛物柱面天线、环柱式天线、双层环形天 线、薄膜天线等最新技术进展进行了介绍, 从构型 设计、优化设计、动力学分析、地面试验等方面阐 述了空间可展开天线机构的研究现状, 从天线与卫 星一体化设计、新材料应用、动力学与控制、试验 验证等方面分析其当前面临的关键问题与发展趋
势。分析表明: 空间可展开天线越来越向超大型化、 超轻量化、高精度方向发展, 需要机械、材料、力 学、控制等多学科交叉融合, 依靠协同创新来推动 可展开天线机构技术的提升。

\section{参 考 文 献}

[1] PELLEGRINO S. Deployable structures[M]. Springer-Verlag Wien, 2001.

[2] 邓宗全. 空间折展机构设计[M]. 哈尔滨：哈尔滨工业 大学出版社, 2013.

DENG Zongquan. Design of space deployable and foldable mechanisms[M]. Harbin: Harbin Institute of Technology Press, 2013.

[3] 郭宏伟, 刘荣强, 李兵. 空间可展开天线机构创新设计 [M]. 北京: 科学出版社, 2018.

GUOHongwei, LIU Rongqiang, LI Bing. Innovative design of space deployable antenna mechanisms[M]. Beijing: Science Press, 2018.

[4] IM E, THOMSON M, FANG H, et al. Prospects of large deployable reflector antennas for a new generation of geostationary Doppler weather radar satellites[C]// AIAA SPACE 2007 Conference \& Exposition. 2007: 1-17.

[5] THOMSON M. AstroMesh deployable reflectors for ku and ka band commercial satellites[C]// 20th AIAA International Communication Satellite Systems Conference and Exhibit. 2002: 2032.

[6] 李团结, 马小飞. 大型空间可展开天线技术研究 [J]. 空 间电子技术，2012，9(3): 35-39.

LI Tuanjie, MA Xiaofei. Technologies of large deployable space antennas[J]. Space Electronic Technology, 2012, 9(3): 35-39.

[7] 刘荣强, 田大可, 邓宗全. 空间可展开天线结构的研究 现状与展望 $[\mathrm{J}]$. 机械设计，2010，27(9): 1-10.

LIU Rongqiang, TIAN Dake, DENG Zongquan. Research actuality and prospect of structure for space deployable antenna[J]. Journal of Machine Design, 2010, 27(9): 1-10.

[8] DATASHVILI L, BAIER H, SCHIMITSCHEK J, et al. High precision large deployable space reflector based on pillow-effect-free technology[C]// 48th AIAA/ASME/AS CE/AHS/ASC Structures, Structural Dynamics, and Materials Conference. 2007: 2186.

[9] MEDZMARIASHVILI E, TSERODZE S, TSIGNADZE $\mathrm{N}$, et al. Earth \& space 2006: Engineering, construction, and operations in challenging environment[M]. U.S.A: ASCE Library, 2006.

[10] MEDZMARIASHVILI E, TSERODZE S, GOGILASHVILI $\mathrm{V}$, et al. Some peculiarities of the creation of deployable ring-shaped space antenna reflectors using flexible rods and ropes[C]// Spacecraft Structures, Materials and 
Mechanical Testing 2005. 2005: 581.

[11] UCHIDA H, OZAWA S, NISHIMOTO M, et al. Buckling mode injection algorithm and its implementation to flexible multibody analysis program[C]// 47th AIAA/AS ME/ASCE/AHS/ASC Structures, Structural Dynamics, and Materials Conference 14th AIAA/ASME/AHS Adaptive Structures Conference 7th. 2006: 2272.

[12] MEguro A, HARAdA S, WATANABE M. Key technologies for high-accuracy large mesh antenna reflectors[J]. Acta Astronautica, 2003, 53(11): 899-908.

[13] 胡飞, 宋燕平, 郑士昆, 等. 空间构架式可展天线研究 进展与展望[J]. 宇航学报, 2018, 39(2): 111-120.

HU Fei, SONG Yanping, ZHENG Shikun, et al. Advances and trends in space truss deployable antennae[J]. Journal of Astronautics, 2018, 39(2): 111-120.

[14] SANTIAGO-PROWALD J, BAIER H. Advances in deployable structures and surfaces for large apertures in space[J]. CEAS Space Journal, 2013， 5(3-4): 89-115.

[15] CAMPBELL B E, HAWKINS W. An 11-METER deployable truss for the seasat radar antenna[C]// Aerospace Mechanisms Symposium. 12th Aerospace Mechanisms Symposium, 1979: 77-88.

[16] LUHMANN H J, ETZLER C C, WAGNER R. Design and verification of mechanisms for a large foldable antenna[C]// Aerospace Mechanisms Symposium. 23rd Aerospace Mechanisms Symposium, 1989: 113-126.

[17] BARTSCH A, TROFAIER A M, HAYMAN G, et al. Detection of open water dynamics with ENVISAT ASAR in support of land surface modelling at high latitudes[J]. Biogeosciences, 2012, 9(2): 703-714.

[18] GRALEWSKI M R, ADAMS L, HEDGEPETH J M. Deployable extendable support structure for the RADARSAT synthetic aperture radar antenna[C]// Washington, DC International Astronautical Federation Congress. 1992.

[19] THOMAS W D R. RADARSAT-2 extendible support structure[J]. Canadian Journal of Remote Sensing, 2004, 30(3): 282-286.

[20] ROSENQVIST A, SHIMADA M, WATANABE M. ALOS PALSAR: Technical outline and mission concepts[C]// 4th International Symposium on Retrieval of Bio-and Geophysical Parameters from SAR Data for Land Applications. Innsbruck, Austria, 2004: 1-7.

[21] NATORI M, TAKANO T, NODA T, et al. Ground adjustment procedure of a deployable high accuracy mesh antenna for space VLBI mission[C]//39th AIAA/ASME/ ASCE/AHS/ASC Structures, Structural Dynamics, and Materials Conference and Exhibit. 1998: 1923.

[22] HANAYAMA E, KURODA S, TAKANO T, et al.
Characteristics of the large deployable antenna on HALCA satellite in orbit[J]. IEEE Transactions on Antennas and Propagation, 2004, 52(7): 1777-1782.

[23] SEMLER D, TULINTSEFF A, SORRELL R, et al. Design, integration, and deployment of the terreStar 18-meter reflector[C]// 28th AIAA International Communications Satellite Systems Conference (ICSSC-2010). 2010: 8855.

[24] GUERCI J, JASKA E. ISAT - innovative space-based-radar antenna technology[C]// IEEE International Symposium on Phased Array Systems and Technology. IEEE, 2003: 45-51.

[25] LANE S A, MURPHEY T W, ZATMAN M. Overview of the innovative space-based radar antenna technology program $[\mathrm{J}]$. Journal of Spacecraft \& Rockets, 2011, 48(1): $135-145$.

[26] FREELAND R E. Survey of deployable antenna concepts[J]. Satellite Communication Antenna Technology, 1983, 1: 613-652.

[27] MEDZMARIASHVILI E , TSERODZE SH , TSIGNADZE N, et al. A new design variant of the large deployable space reflector[C]// The 10th Biennial International Conference on Engineering, Construction, and Operations in Challenging Environments. Houston, TX, United States: ASCE, 2006: 1-8.

[28] DAI L, GUAN F, GUEST J K. Structural optimization and model fabrication of a double-ring deployable antenna truss[J]. Acta Astronautica, 2014, 94(2): 843-851.

[29] XU Y. Structural design and static analysis of a double-ring deployable truss for mesh antennas[J]. Acta Astronautica, 2012, 81(2): 545-554.

[30] GILGER D L, PARKER D A. Folding perimeter truss reflector: USA[P]. 2000-02-22.

[31] 肖潇. 空间薄膜结构的展开动力学研究及热分析 [D]. 杭州: 浙江大学, 2009.

XIAO Xiao. Deployment dynamics research and thermal analysis for space membrane[D]. Hangzhou: Zhejiang University, 2009.

[32] LEIPOLD M, WIDANI D, GROEPPER P. The European solar sail deployment demonstration mission[C]// 57th International Astronautical Congress, Valencia, Spain, 2006, IAC-06-A3.4.07

[33] COPP T, DOMBER J L, ATCHESON P D, et al. MOIRE: Membrane material property characterizations, testing and lessons learned[C]// Spacecraft Structures Conference. 2013.

[34] 谢军. 充气膜结构的褶皱及振动特性研究[D]. 哈尔滨: 哈尔滨工业大学, 2012.

XIE Jun. Study on fold and vibration characteristics of inflatable membrane structure[D]. Harbin: Harbin Institute of Technology, 2012. 
[35] 成新兴. 空间充气可展硬化薄膜天线结构热分析[D]. 上海：上海交通大学, 2011.

CHENG Xinxing. Thermal analysis of structure of space inflatable expandable hardened thin film antenna[D]. Shanghai: Shanghai Jiao Tong University, 2011.

[36] 谢军. 充气膜结构的褶皱及振动特性研究 [D]. 哈尔滨: 哈尔滨工业大学, 2012.

XIE Jun. Study on fold and vibration characteristics of inflatable membrane structure[D]. Harbin: Harbin Institute of Technology, 2012.

[37] 毕朕. 空间充气管展开过程仿真及模态分析[D]. 哈尔 滨: 哈尔滨工业大学, 2006.

BI Zhen. Simulation and modal analysis of space inflatable pipe expansion process[D]. Harbin: Harbin Institute of Technology, 2006.

[38] 徐彦. 充气可展开天线精度及展开过程分析研究[D]. 杭州: 浙江大学, 2009 .

$\mathrm{XU}$ Yan. Analysis and research on the accuracy and expansion process of inflatable expandable antenna[D]. Hangzhou: Zhejiang University, 2009.

[39] 姜伟. 空间充气可展天线反射面设计、分析与试验研究 [D]. 上海：上海交通大学，2007.

JIANG Wei. Design, analysis and experimental study of reflective surface of space inflatable expandable antenna[D]. Shanghai: Shanghai Jiao Tong University, 2007.

[40] PELLEGRINO S, YOU Z. Foldable-bar-structures[J]. International Journal of Solids and Structures, 1997, 34(15): 1825-1847.

[41] KASSABIAN P E, YOU Z, PELLEGRINO S. Retractable roof structures[J]. Proceedings of Institution of Civil Engineers Structures and Building, 1999, 134: 45-56.

[42] PATEL J, ANANTHASURESH G K. A kinematic theory for radially foldable planar linkages[J]. International Journal of Solids and Structures, 2007, 44(18-19): 6279-6298.

[43] TANAKA H. Planar mobility modes of 8-bar-jointed structures with a single degree of freedom[J]. International Journal of Solids and Structures, 2012，49(13): 1712-1722.

[44] WARNAAR D B, CHEW M. Kinematic synthesis of deployable-foldabletruss structure using graph theory part 1: Graph generation[J] .Transaction of the ASME, Journal of Mechanical Design, 1995, 117: 112-116.

[45] WARNAAR D B, CHEW M. Kinematic synthesis of deployable-foldabletruss structure using graph theory part 2: Generation of deployable truss module design concepts[J]. Transaction of the ASME, Journal of Mechanical Design, 1995, 117: 117-122.

[46] WOHLHART K. Regular polyhedral linkages[C]//
Proceedings of the Second Workshop on Computational Kinematics 2001, Seoul, Korea, 2001: 239-248.

[47] KOVACS F. A class of expandable polyhedral structures[J]. International Journal of Solids and Structures, 2004, 41(3-4): 1119-1137.

[48] HOBERMAN C. Geared expanding structures: USA, US7464503B2[P]. 2004-07-02.

[49] GOSSELIN C M. Expandable polyhedral mechanisms based on polygonal one-degree-of-freedom faces[J]. Proceedings of the Institution of Mechanical Engineers, Part C: Journal of Mechanical Engineering Science, 2006, 220(7): 1011-1018.

[50] KIPER G. Polyhedral linkages synthesized using Cardan motion along radial axes[C]// Pro. 12th World Cong. Theory of Mach. Mech. France, 2007: 471-477.

[51] KIPER G. A family of deployable polygons and polyhedral[J]. Mechanism and Machine Theory, 2008, 43(5): $627-640$.

[52] CHEN Y. Threefold-symmetric Bricard linkages for deployable structures[J]. International Journal of Solids and Structures, 2005, 42(8): 2287-2301.

[53] YOU Z, CHEN Y. Mobile assemblies based on the Bennett linkage[J]. Proceedings of the Royal Society A: Mathematical, Physical and Engineering Sciences, 2005, 461: 1229-1245.

[54] YOU Z, CHEN Y. Square deployable frames for space applications. Part 1: Theory[J]. Proceedings of the Institution of Mechanical Engineers, Part G: Journal of Aerospace Engineering, 2006, 220: 347-354.

[55] CHEN Y, YOU Z. Spatial 6R linkages based on the combination of two Goldberg 5R linkages[J]. Mechanism and Machine Theory, 2007, 42(11): 1484-1498.

[56] YOU Z, CHEN Y. Square deployable frames for space applications. Part 2: Realization[J]. Proceedings of the Institution of Mechanical Engineers, Part G: Journal of Aerospace Engineering, 2007, 221(1): 37-45.

[57] CHEN Y, YOU Z. On mobile assemblies of Bennett linkages $[\mathrm{J}]$. Proceedings of the Royal Society A : Mathematical, Physical and Engineering Sciences, 2008, 464(2093): 1275-1293.

[58] CHEN Y, YOU Z. Two-fold symmetrical 6R foldable frame and its bifurcations[J]. International Journal of Solids and Structures, 2009, 46(25-26): 4504-4514.

[59] CHEN Y, YOU Z. An extended myard linkage and its derived 6R linkage[J]. Journal of Mechanical Design, 2008, 130(5): 052301-1-8.

[60] GAN W W, PELLEGRINP S. Numerical approach to the kinematic analysis of deployable structures forming a closed loop[J]. Proceedings of the Institution of Mechanical Engineers, Part C: Journal of Mechanical Engineering 
Science, 2006, 220(7): 1045-1056.

[61] BAKER J E. On generating a class of foldable six-bar spatial linkages[J]. Journal of Mechanical Design, 2006, 128(2): 374-383.

[62] BAKER J E. Kinematic investigation of the deployable Bennett loop[J]. Journal of Mechanical Design, 2007, 129(6): 602-610.

[63] VIQUERAT A D. A plane symmetric 6R foldable ring[J]. Mechanism and Machine Theory, 2013, 63: 73-88.

[64] YU Y. Deployable membrane structure based on the Bennett linkage[J]. Proceedings of the Institution of Mechanical Engineers, Part G: Journal of Aerospace Engineering, 2007, 221(5): 775-783.

[65] NAGASE K, SKELTON R E. Minimal mass tensegrity structures[J]. Journal of the International Association for Shell and Spatial Structures, 2014, 55(1): 37-48.

[66] NAGASE K, SKELTON R E. Minimal mass design of tensegrity structures[C]// Sensors and Smart Structures Technologies for Civil, Mechanical, and Aerospace Systems 2014. International Society for Optics and Photonics, 2014: 90610W.

[67] SKELTON R E, FRATERNALI F, CARPENTIER G, et al. Minimum mass design of tensegrity bridges with parametric architecture and multiscale complexity[J]. Mechanics Research Communications, 2014, 58: 124-132.

[68] 尤国强. 周边桁架可展开天线的结构优化设计[D]. 西 安: 西安电子科技大学, 2005 .

YOU Guoqiang. Structural optimization of flexible cable net deployable antenna[D]. Xi'an: Xidian University, 2005.

[69] 李涁. 网状反射面天线的结构优化设计研究[D]. 西安: 西安电子科技大学, 2010 .

LI Bin. Study on structural optimization design of mesh deployable antennas[D]. Xi'an: Xidian University, 2005.

[70] 罗鹰. 大型星载可展开天线的动力优化设计与工程结 构的系统优化设计 [D]. 西安: 西安电子科技大学, 2004.

LUO Ying. Study on dynamic optimization of large deployed antenna and structural systematic optimization[D]. Xi'an: Xidian University, 2004.

[71] FANG H, LOU M, HSIA L, et al. Catenary systems for membrane structures[J]. AIAA paper, 2001, 1342: 1.

[72] SAKAMOTO H. Dynamic wrinkle reduction strategies for membrane structures[M]. 2004.

[73] GRESCHIK G, PALISOC A, CASSAPAKIS C, et al. Sensitivity study of precision pressurized membrane reflector deformations[J]. Aiaa Journal, 2015, 39(2): 308-314.

[74] SOH M, LEE J H, YOUN S K. An inflatable circular membrane mirror for space telescopes[C]// Photonics Asia.
International Society for Optics and Photonics, 2005: 262-271.

[75] 关富玲, 侯国勇, 赵孟良. 构架式可展开天线结构设计 的程序实现 [J]. 工程设计学报, 2006, 12(2): 108-113. GUAN Fuling, HOU Guoyong, ZHAO Mengliang. Program implementation of structure design for deployable truss antenna[J]. Journal of Engineering Design, 2006, 12(2): 108-113.

[76] SHABANA A A. An absolute nodal coordinates formulation for the large rotation and deformation analysis of flexible bodies[R]. University of Illinois at Chicago: MBS96-1-UIC, 1996.

[77] QUISENBERRY J E, ARENA A S. Discrete cable modeling and dynamic analysis[C]// 44th AIAA Aerospace Sciences Meeting and Exhibit. Reno, Nevada, 2006: 424.

[78] POOLE L R, HUCKINS E K. Evaluation of massless-spring modeling of suspension-line elasticity during the parachute unfurling process[R]. Washington: NASA, 1972.

[79] MITSUGI K A, SENBOKUYA Y, MEGURO A. Deployment analysis of large space antenna using flexible multibody dynamics simulation[J]. Acta Astronautica, 2000, 47(1): 19-26.

[80] NETO M A, LEAL R P. Composite materials in flexible multibody systems[J]. Computer Methods in Applied Mechanics and Engineering, 2006, 195(50-51) : 6860-6873.

[81] 虞碟, 任革学. 基于绝对坐标的实体单元在多体系统动 力学中的应用 [J]. 系统仿真学报, 2012, 24(3): 733-739. YU Lei, REN Gexue. Application of solid elements using absolute coordinates in multibody systems dynamics[J]. Journal of System Simulation, 2012，24(3): 733-739.

[82] 刘铖, 田强, 胡海岩. 基于绝对节点坐标的多柔体系统 动力学高效计算方法 $[\mathrm{J}]$. 力学学报, 2010, 42(6): 1197-1205.

LIU Cheng, TIAN Qiang, HU Haiyan. Efficient computational method for dynamics of flexible multibody systems based on absolute nodal coordinate[J]. Chinese Journal of Theoretical and Applied Mechanics, 2010, 42(6): 1197-1205.

[83] 蒋建平. 大挠性多体结构卫星刚柔耦合动力学研究[D]. 长沙: 国防科学技术大学, 2004 .

JIANG Jianping. Researches on rigid-flexible coupling dynamics of the flexible multibody satellite[D]. Changsha: National University of Defense Technology，2004.

[84] 刘钦鹏, 段宝岩, 杨东武. 柔性空间展开机构动力学建 模研究 [J]. 机械设计, 2006, 23(3): 1-4.

LIU Qinpeng, DUAN Baoyan, YANG Dongwu. Study on the dynamics modeling of development mechanism in flexible space[J]. Journal of Machine Design, 2006, 
23(3): $1-4$.

[85] LIU C, TIAN Q, HU H Y. Dynamics of a large scale rigid-flexible multibody system composed of composite laminated plates[J]. Multibody System Dynamics, 2011, 26(3): 283-305.

[86] 齐乃明, 张文辉, 高九州, 等. 空间微重力环境地面模 拟试验方法综述 [J]. 航天控制, 2011，29(3): 95-100. QI Naiming, ZHANG Wenhui, GAO Jiuzhou, et al. The primary discussion for the ground simulation system of spatial microgravity[J]. Aerospace Control, 2011, 29(3): 95-100.

[87] 姚燕生. 三维重力补偿方法与空间浮游目标模拟实验 装置研究[D]. 合肥: 中国科学技术大学, 2006 .

YAO Yaosheng. Research on 3-D gravity compensation and equipment of space floating objective simulation[D]. Hefei: University of Science and Technology of China, 2006.

[88] 张孝谦, 袁龙根, 吴文东, 等. 国家微重力实验室百米 落塔实验设施的几项关键技术 $[\mathrm{J}]$. 中国科学 $\mathrm{E}$ 辑: 工程 科学, 材料科学, 2015, 35(5): 523-534.

ZHANG Xiaoqian, YUAN Longgen, WU Wendong, et al. Several key technologies for NMLC's 100-meter drop tower experimental facility[J]. Science in China Ser. E, Engineering \& Materials Science, 2015, 35(5): 523-534.

[89] 高海波, 郝峰, 邓宗全, 等. 空间机械臂收拢状态零重 力模拟 [J]. 机器人, 2011, 33(1): 9-15.

GAO Haibo, HAO Feng, DENG Zongquan, et al. Zero-g simulation of space manipulator in furled status[J]. Robot, 2011, 33(1): 9-15.

[90] GEFKE G G, CARIGNAN C R, ROBERTS B J, et al. Ranger telerobotic shuttle experiment: Status report[C]// Proceedings of SPIE, Bellingham, WA, USA: SPIE, 2001: 123-132.

[91] TSUNODA H, HARIU K, KAWAKAMI Y, et al. Deployment test methods for a large deployable mesh reflector[J]. Journal of Spacecraft and Rockets, 1997, 34(6): 811-816.

[92] TSUNODA H, HARIU K, KAWAKAMI Y, et al. Structural design and deployment test methods for a large deployable mesh reflector[C]// AIAA /ASME/ ASCE / AHS /ASC Structures, Structural Dynamics and Materials Conference. Kissimmee, FL, USA: AIAA, 1997 : 2963-2971.

[93] MEFURO A, ISHIKAWA H, TSUJIHATA A. Study on ground verification for large deployable modular structures[J]. Journal of spacecraft and rockets, 2006, 43(4): 780-787.

[94] 韦娟芳. 空间 4 10 米可展开天线的动力耦合分析及 实验技术[D]. 杭州: 浙江大学, 2002.
WEI Juanfang. Dynamic interaction analysis of space 4-10 $\mathrm{m}$ deployable antenna and its test technics[D]. Hangzhou: Zhejiang University, 2002.

[95] 张慧峰. 空间可展开天线精度测量、热分析、可靠性 分析及间隙影响研究[D]. 杭州：浙江大学, 2010 .

ZHANG Huifeng. Surface precision measurement, space thermal, reliability and clearance effect analysis of deployable space antennas[D]. Hangzhou: Zhejiang University, 2010.

[96] 吴建云, 王春洁, 汪瀚. 基于蒙特卡洛法的卫星天线 板展开精度分析[J]. 航天返回与遥感，2013，34(6): 89-94.

WU Jianyun, WANG Chunjie, WANG Han. Accuracy analysis of satellite antenna plate deployment based on Monte Carlo method[J]. Spacecraft Recovery \& Remote Sensing, 2013, 34(6): 89-94.

[97] 丁建中，王春洁. 含铰链间隙板式卫星天线展开精度 分析 [J]. 北京航空航天大学学报, 2016, 42(12): 2625-2631.

DING Jianzhong, WANG Chunjie . Deployment accuracy analysis of planar satellite antenna with joint clearances[J]. Journal of Beijing University of Aeronautics and Astronautics, 2016, 42(12): 2625-2631.

[98] 张俊刚, 何玲, 高海洋, 等. 大型航天器动力学试验 验证技术进展 $[\mathrm{J}]$. 中国科学: 物理学 力学 天文学, 2019, 49(2): 024507.

ZHANG Jungang, HE Ling, GAO Haiyang, et al. Development of the method of the large scale spacecraft dynamic environment test[J]. Scientia Sinica Pysica, Mechanica \& Astronomica, 2019, 49(2): 024507.

[99] MISAWA M, OGAWA A. Analytical and experimental frequency verification of deployed satellite antennas $[\mathrm{C}] / /$ 44th AIAA/ASME/ASCE/AHS Structures, Structural Dynamics, and Materials Conference. Norfolk, Virginia: AIAA, 2003: 879-885.

[100] ANDO K, MITSUGI J, SENBOKUYA Y. Analyses of cable-membrane structure combined with deployable truss[J]. Computers and Structures, 2000， 74(1): 21-39.

[101] 田大可. 模块化空间可展开天线支撑桁架设计与实验 研究[D]. 哈尔滨：哈尔滨工业大学，2011.

TIAN Dake. Design and experimental research on truss structure for modular space deployable antenna[D]. Harbin: Harbin Institute of Technology, 2011.

作者简介: 刘荣强, 男, 1965 年出生, 博士, 教授, 博士研究生导师。 主要研究方向为空间大型可展开机构与控制。

E-mail: liurq@hit.edu.cn 郭宏伟(通信作者), 男, 1980 年出生, 博士，副教授，博士研究生导师。 主要研究方向为空间可展开机构与控制与可变构型结构与机构。 E-mail: guohw@hit.edu.cn 